\title{
Service-Oriented Middleware Architecture for Mobile Personal Health Monitoring
}

\author{
Matts Ahlsén, Stefan Asanin, Peeter Kool, Peter Rosengren \\ CNet Svenska AB, Svärdvägen 3b, 18233 Danderyd, Sweden \\ \{matts.ahlsen, stefan.asanin, peeter.kool, peter.rosengren\}@cnet. se \\ Jesper Thestrup \\ In-JeT ApS, Jeppe Aakjaers Vej 15, 3460 Birkeroed, Denmark \{jth@in-jet.dk\}
}

\begin{abstract}
Developers of applications for health and wellness monitoring are facing a diversity of protocols, standards and communication mechanisms for collecting data from heterogeneous sensors, devices and services, as well as when exporting data to various health and wellness services and systems. The REACTION platform addresses this using a middleware approach which leverages the development tasks to a service-oriented level allowing developers to use open standard technologies like web services. The REACTION SOA (Service-Oriented Architecture) approach offers a scalable and interoperable platform for use in different healthcare settings. The REACTION applications are based on numerous individual services that can be developed and deployed to perform clinical monitoring and feedback tasks, execute distributed decision support and security tasks, support work flow management, and perform event handling and crisis management.
\end{abstract}

Keywords: Remote Healthcare Monitoring, Diabetes, SOA, Internet of Things, Middleware, Semantics.

\section{Introduction}

The REACTION project [1] aims to research and develop an intelligent service platform that can provide professional, remote monitoring and therapy management to diabetes patients in different healthcare regimes across Europe. The platform is designed to help developers create support for both carers and patients in the management of diabetes but has the possibility of managing other chronic diseases. Today developers of applications for health and wellness monitoring are facing a diversity of protocols, standards and communication mechanisms both when collecting data from heterogeneous sensors, devices and services, as well as when exporting data to various health and wellness services and systems. For example, IEEE11073 standards are being used by the Continua Alliance [14], while there are numerous legacy devices using proprietary interfaces both over Bluetooth as well as through USB or serial port. In the wellness sector there are many ANT+ [5] sensors available, and recently several WIFI-based health devices [8] with associated Internet 
services have been introduced. In addition to this several cloud-based health services offers services for uploading and analysing the collected health data [7], [13].

The REACTION platform addresses this heterogeneity by applying an "Internet of Things" perspective on medical device connectivity. It uses a middleware approach which leverages the developer's tasks to a service-oriented level allowing developers to use open standard technologies like web services. The middleware approach also makes the applications independent of the underlying device and service protocol level and ensures interoperability as well as re-usability, since new devices can be deployed and/or old ones replaced without the applications have to be re-built.

The REACTION middleware builds on results from the integrated EU project Hydra which researched Open Source middleware for Internet of Things. The Hydra middleware incorporates support for ontology-driven discovery of devices, P2P (Peerto-Peer) communications, use of semantic technologies for code generation [2],[4],[11]. The REACTION project extends and adapts the middleware to device connectivity in the health and wellness sectors. This allows developers to rapidly create health and wellness applications as collections of services which can be orchestrated to perform desired workflows supported through the platform spheres (Fig. 1), which improves development efficiency while delivering trusted and reliable patient-oriented services.

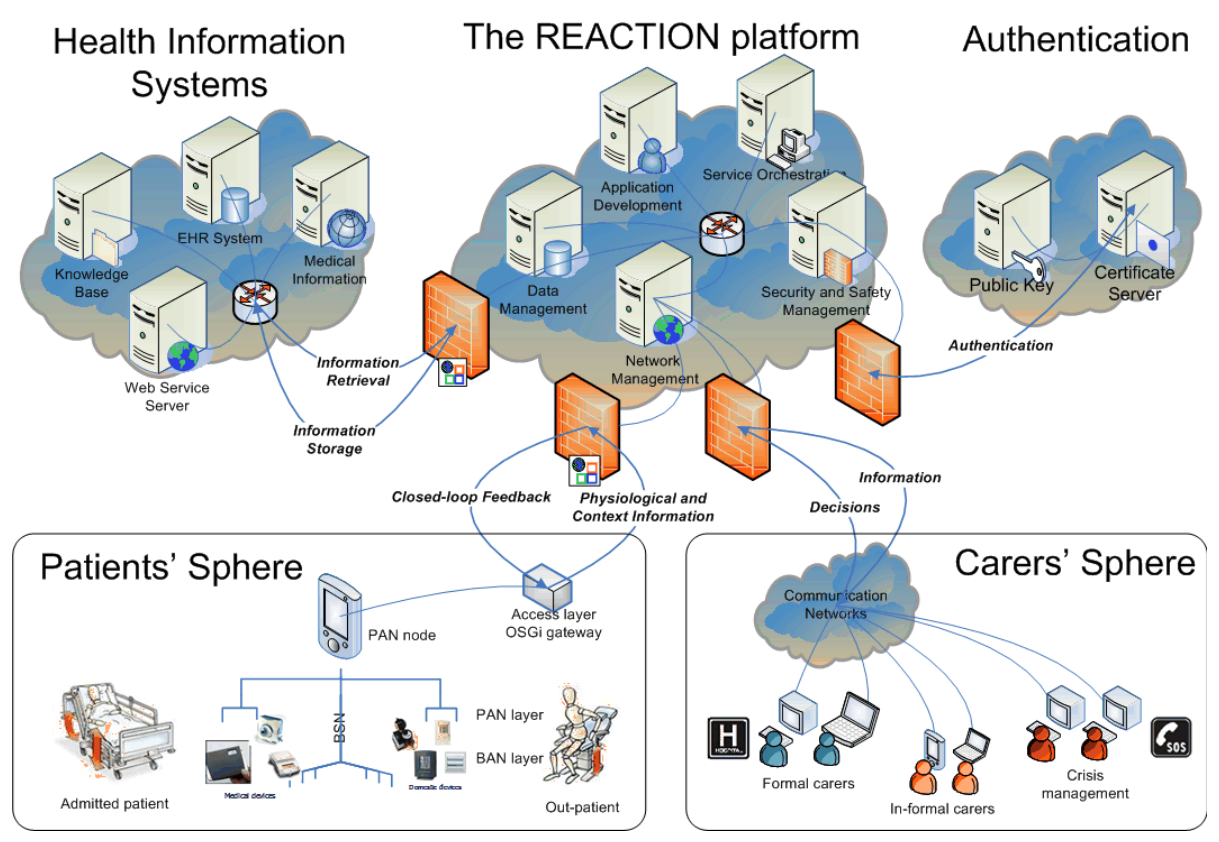

Fig. 1. The REACTION platform concept with two sphere (patient and carer) depicting the dynamics of a SOA-based framework for remote patient monitoring. 
Wearable medical sensors are connected in a BAN (Body Area Network) for multiparametric recording of vital physiological parameters. The BAN interconnects with other sensors in the environment that can record contextual information about other vital parameters and the patients' activities in a PAN (Personal Area Network). A local REACTION gateway also handles episode monitoring, alarms and services needed during periods of non-connectivity. The gateway further manages personalised patient feedback from health professionals adapted to user terminals and self-monitoring and autonomous regulation of the connected devices in the BAN. The gateway can be remotely configured and managed through the service-oriented architecture.

Health Information Systems (HIS) will be integrated in REACTION applications in the form of services, these services can subsequently be orchestrated into workflows through the Service Orchestration subset.

\section{Usage Scenarios}

This chapter describes some typical monitoring applications that could be built using the REACTION platform. The purpose is to illustrate the use of different technologies in a chronic disease management perspective. We assume that the patient is at home and REACTION is integrated in the home environment. The first two scenarios take part as diabetes' co-morbidities.

Heart failure The application developed monitors the patient with ECG (Electrocardiogram), using a wireless electronic patch, ePatch [15], which is part of the REACTION project. This ePatch contains sensors and a ZigBee chip. The patch transmits data to the REACTION gateway hosting the REACTION client software. The data is rendered in a user interface to allow clinicians to analyse it. The ePatch allows the user to move freely around all day. The patient has also been advised to exercise, so he makes long walks every day. Using an ANT+ enabled GPS-watch he can then upload the time and length of each walk into an account in a personal cloud service, like HealthVault [7]. Together with the watch he sometimes uses a heart rate monitor attached to his breast to measure the pulse. Monitoring hypertension may also help the care of diabetes patients.

Obesity:A Bluetooth-enabled weight scale based on the IEEE 11073 [6] standard is installed in the bathroom. Every morning the patient is reminded by the REACTION application running in his smart phone to perform the weight measurement. The patient steps on the weight scale and the measured weight is then automatically transferred to a stationary or portable PC in the home. The patient has an account with a cloud based health service, like WiThings [8], which he uses to keep track of his weight to see if there is any progress in losing weight with the new exercise and diet program he is using. The REACTION software connects to the cloud health service, using credentials the patient has provided at the time of set up. All diabetic patients also have dietetic issues and so monitoring weight may assist in personal satisfaction and disease management. 
Diabetes: The patient has been ordered to measure his blood pressure and glucose level every evening. The reaction platform is able to discover and interface to all devices used in the application, including Continua certified.

Unlike weight measurements the blood pressure and monitored glucose levels are exported to a primary care centre after being aggregated by REACTION data fusion engine together with data related to food intake, e.g. carbohydrates. The blood pressure monitor he has is not Continua-certified. He measures his blood pressure sitting in the living room sofa, while watching TV. The blood pressure values are received by the REACTION client in the same way as the weight in previous, but the values are exported to a primary care centre, where a REACTION server receives the values and stores it in the care centers EPR. In addition to this, the patient has, for his personal interest, configured REACTION to export the blood pressure values to a health service, HealthVault.

The most critical vital sign for the patient is the glucose, which requires him to use a glucometer. This is a cheaper model that is not wireless but attaches to the USB-port of the computer. He uses a blood sugar test strip which is inserted into the USB-based glucose meter. The glucose values are collected by REACTION and transmitted to the primary care centre. All communication with the remote server is done using IHEPCD formats [16]. The glucose values are not transmitted to cloud services since the patient has defined a security policy, by which these values cannot be exported anywhere else except to the primary care centre. But before sending the glucose values, REACTION also performs data fusion and attaches information related to the patient's food intake this day, such as the level of carbohydrates, etc. This has been manually entered by the patient during the day. REACTION also adds today's weight before everything is sent to the primary care database. If the patient forgets to take his blood pressure and/or glucose level, REACTION will interface with other devices at home, in order to notify the patient or carers by means of an alarm or messaging function specific to the situation at hand. This is configurable by setting up rules which are executed by a rule engine. In fact, it is possible for REACTION, due to its P2P service-oriented architecture, to connect to other homes, for instance the home of a relative and alert them of that something went wrong at the patient's home.

\section{Service-oriented Middleware}

The REACTION platform is the central production environment for the deployment of REACTION applications consisting of five subsets providing different functionalities:

- Data Management implements data manipulation, data fusion [3], event handling and data transport.

- Service Orchestration orchestrates available services in a pre-described sequence for execution.

- Network Management is responsible for the communication between devices, persons and external repositories.

- Security Management manages security models over user devices. 
- Application Development is an open SDK (Software Development Kit) for model-driven development of applications. Context awareness is achieved through semantic annotations from patients' devices, environment and from historical data in EPRs (Electronic Patient Records).

The REACTION platform architecture (Fig. 2) is component based. Components relevant for the SOA approach are described in the following subchapters.

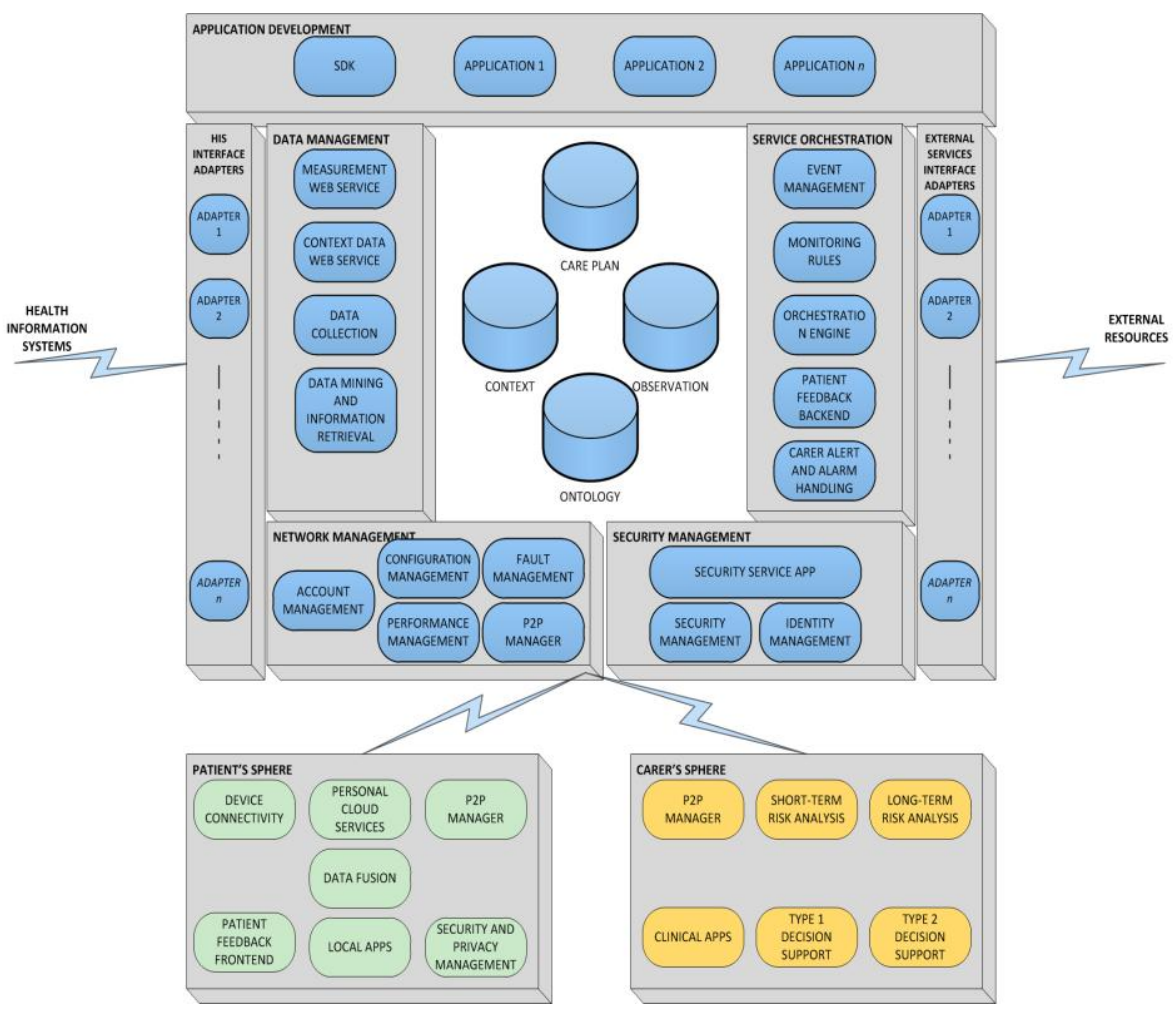

Fig. 2. The REACTION platform with generic service components where the application level provides an orchestration mechanism for design of end-user applications.

\section{Medical Device Connectivity Kit}

The service-oriented middleware is made available to developers through a Medical Device Connectivity Kit (DCK) that enables developers to rapidly and seamlessly integrate medical Continua (i.e. IEEE 11073), medical non-Continua and wellness devices (e.g. exercise machines) into any development environment by the use of XML and web services. Using the DCK a developer can support traditional pushbased remote patient monitoring applications, where a device pushes a measurement 
through to an observations database on the clinic side, local client applications that renders data to the user either in the home or as an app in a smart phone and clinical applications to analyse collected data.

Fig. 3 shows the internal structure of the DCK. In the bottom a Protocol Module ensures that the local environment is searched for both new and existing devices and whenever a Health Device Profile (HDP) exists for a device, the module is able to communicate with it over a low level radio.

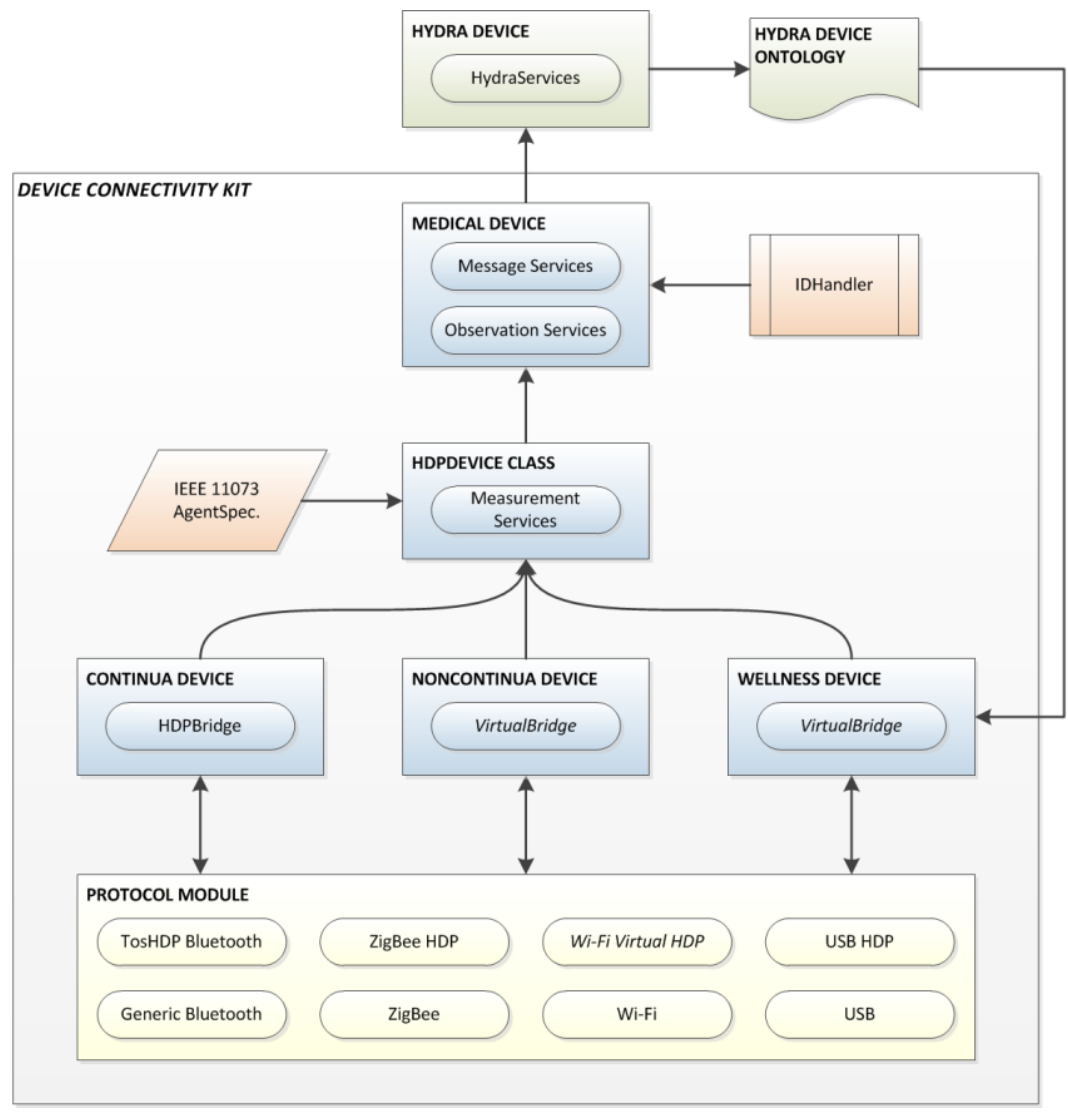

Fig. 3. The HDPDevice class holds Measurement Services that can either use the IEEE 11073 Agent Specialisations or relate the services to those described in the Device Ontology.

In cases where no matching HDP exists, the Protocol Module will provide a virtual device profile. . This enables the communication between the module and the bridge in the different device types. Each device type announces what type of sensor device is trying to communicate whereas the HDPDevice compares this information to the IEEE 11073 specialisations available in a library. If such device exists as part of the standard, the HDPDevice will establish a wide range of characteristics relevant for this sensor device type and enable a push approach, whereby the sensor data are forwarded as part of a Measurement Service. 
On the top, the super class Medical Device sets parameters such as patient ID and therapy related data through the IDHandler. This helps in constructing a complete HL7 (Health Level Seven) message based on the ORU-R01 format [12]. Finally, the Hydra Device class allows all underlying functionalities and services to be made available through Web Services.

\section{Data Fusion and Service Orchestration}

The REACTION applications are based on numerous individual services that can be developed and deployed to perform clinical monitoring and feedback tasks, execute distributed decision support and security tasks, support work flow management, and perform event handling and crisis management.

SOA is a collection of services that communicate with each other. The aim of a SOA approach is to have interoperable and loosely coupled services distributed in the network. In this context, a service is a function that is well-defined, self-contained, and does not depend on the context or state of other services.

In the REACTION platform, each device is enabled to offer Web Services that can be consumed by other devices, services or applications through an overlying mobile P2P network. Every service offered by a physical device is identified and invoked using SOAP (Simple Object Access Protocol) messages that are transmitted over the P2P network to create a robust connection, and WSDL (Web Service Definition Language) to define the interface of the services, no matter the implementation language used.

Ensembles of REACTION services are orchestrated by a specific high-level workflow based on BPEL (Business Protocol Execution Language). The workflow will be specified in the application and interpreted by the Orchestration Manager. The Orchestration Manager will make sure that the different available services are executed in the described sequence. This component introduces higher abstraction mechanisms and makes the application developer independent of using a specific programming environment to orchestrate REACTION applications. It will also eliminate the interdependencies of services, solve conflicts of services and provide the most flexibility environment needed to realise service-oriented applications.

\section{Conclusion and Future work}

In this paper we have described how a service-oriented middleware approach can be employed to facilitate development of health and wellness applications allowing semantic interoperability of heterogeneous devices, services and applications. Specifically, REACTION provides an integrated development and run-time environment for the improved long-term management of diabetes and other chronic diseases. We have explained how tasks such as connecting and collecting values from a medical devices, data fusion from many sensors, service orchestration, export of medical data can be realised using a service-oriented approach. A Medical Device Connectivity Kit will make the middleware functionality available for developers, and allow creation of applications based on open standards. 


\section{Acknowledgment}

This work was performed in the framework of FP7 Integrated Project REACTION (Remote Accessibility to Diabetes Management and Therapy in Operational Healthcare Networks) partially funded by the European Commission. The authors wish to express their gratitude to the members of the REACTION consortium for valuable discussions.

\section{References}

1. REACTION Project, http://reaction-project.eu/

2. Eisenhauer, M., Rosengren, P., Antolin, P.: A Development Platform for Integrating Wireless Devices and Sensors into Ambient Intelligence Systems. In: Proceedings of the 6:th Annual IEEE Communications Society Conference on Sensor, Mesh and Ad Hoc Communications and Networks (SECON), pp. 1--3. IEEE Press, Rome, Italy (2009)

3. Lee, H., Park, K., Choi, J., Lee, B., Elmasri, R.: Issues in Data Fusion for Healthcare Monitoring. In: PETRA'082008. ACM, Athens, Greece (2008)

4. Hydra Project, http://www.hydramiddleware.eu/

5. ANT+, http://www.thisisant.com/pages/technology/what-is-ant-plus

6. Carroll, R., Cnossen, R., Schnell, M., Simons, D.: Continua: An Interoperable Personal Healthcare Ecosystem. In: IEEE Pervasive Computing, Mobile and Ubiquitous Systems, 6, pp. 90--94 (2007)

7. Microsoft HealthVault, www.healthvault.com

8. Withings, http://www.withings.com

9. Bluetooth SIG, http://www.bluetooth.com

10. ZigBee Alliance, http://www.zigbee.org

11. Lardies, F.M., Antolin, P., Fernandes, J., Zhang, W., Hansen, K., Kool, P.: Deploying Pervasive Web Services over a P2P Overlay. In: 18th IEEE International Workshops on Enabling Technologies: Infrastructures for Collaborative Enterprises, pp. 240--245. IEEE Computer Society, Groningen, The Netherlands (2009)

12. Health Level Seven, http://www.hl7.org

13. HealthGraph, http://developer.runkeeper.com/healthgraph/overview

14. Continua Alliance, http://www.continuaalliance.org

15. ePatch, http://www.madebydelta.com/delta/Business_units/ME/Body_sensors/ePatch.page

16. IHE, http://www.ihe.net 